\title{
Velogenic newcastle disease virus tissue tropism and pathogenesis of infection in chickens by application of in situ PCR, immunoperoxase staining and HE staining
}

\begin{abstract}
Limited deep studies are available in the field of early stages of pathogenesis of Newcastle disease virus (NDV) infection and tissue tropism of NDV. In this study, 24 specific pathogen free (SPF) chickens of white leghorn breed were infected with Newcastle disease (ND) by intranasal administration of $10750 \%$ EID50/0.1 mL of velogenic NDV (vNDV). A second group of 15 chickens were kept as a control group. Chickens were monitored every day to record clinical signs. Infected chickens were euthanized by cervical dislocation at successive times, namely at hours (hrs) 2, 4, 6, 12, days 1, 2, 4, and 6 post-inoculation (pi). Whereas, control group chickens were euthanized on days $0,1,2,4$, and 6 pi. Tissues of brain, trachea, lung, caecal tonsil, liver, kidney, spleen, heart, proventriculus, intestine, and thymus were collected, fixed in $10 \%$ buffered formalin, embedded in paraffin, and sectioned. HS staining, immunoperoxidase staining (IPS) and in situ PCR were applied. It was concluded that at hr 2 pi, virus seemed to be inclined to trachea and respiratory tract. Meanwhile, it attacked caecal tonsils, intestine and bursa of Fabricus. While primary viraemia was ongoing, virus created footing in kidney and thymus. At hr 4 pi, proventriculus, liver, and spleen were attacked. However, at hr 6 pi, brain and heart were involved. Secondary viraemia probably started as early as hr 12 pi since all collected tissues were positive. Tissue tropism was determined in trachea, caecal tonsil, liver, bursa of Fabricius, intestine, proventriculus, lung, spleen, thymus, kidney, heart, and brain.
\end{abstract}

Keyword: In situ PCR; Velogenic newcastle disease virus; Pathogenesis; Tissue tropism 